\title{
Laser generation and detection of SAW at the surface of PMMA
}

\author{
X. Zhang, C. Gan, D. Fei and S. Zhang \\ Institute of Acoustics, Laboratory of Modern Acoustics, Nanjing University, Nanjing 210008, China
}

\begin{abstract}
The SAW velocity of PMMA as a function of temperature $\left(25-75^{\circ} \mathrm{C}\right)$ and frequency $(1.0-8.0 \mathrm{MHz})$ is measured by the laser generation and detection technique. From the temperature dependence of the velocity two turning points are observed, one of which may be related to the $\beta$-transition of PMMA.
\end{abstract}

\section{INTRODUCTION}

The polymethyl methacrylate(PMMA) may be considered as a typical amorphous polymer, which is viscoelastic. For most of the polymers, they are almost perfectly elastic solids at low enough temperatures, but change to viscous liquids at high temperatures, and exhibit viscoelasticity in the intermediate range. The viscoelastic properties are of great importance in materials characterization and nondestructive evaluation(NDE). Several researchers[1-3] have used the ultrasonic NDE technique to characterize polymers. Their results showed that the stress relaxation function or complex modules of polymers can be evaluated from the measurements of velocities and absorptions of the bulk waves as a function of frequency and temperature. The properties of SAW propagating along the surface of polymer are also important. But in our knowledge, they have not been known so far.

In principle, the viscoelastic wave motion equation can be derived from the elastic wave motion equation and by taking the delayed elasticity into account. Then the velocity of SAW can be deduced from the dispersive equation. However, it is always very complicated and has no analytical solution. On the other hand, most of the polymers are nonpiezoelastic, it becomes difficult to excite and detect the SAW by usual ultrasonic method for small samples. While in a laser ultrasonic system, the SAW can be generated and detected by laser technique, hence the SAW velocity can be easily measured. Besides, it has the advantages of non-contact, nondestructive and flexible source size, which are suitable for the investigation of the properties of acoustic bulk waves and SAW[4]. In this article, the laser ultrasonic technique used to generate and detect SAW at the surface of PMMA sample is described. The result shows that there are two turning points in the temperature dependence of the SAW velocity from $25^{\circ} \mathrm{C}$ to $75^{\circ} \mathrm{C}$, one of which is related to the $\beta$-transition of PMMA. The frequency dependence of the SAW velocity for PMMA is also given.

\section{BASIC IDEA}

When a laser pulse is focused onto the surface of a PMMA sample (see Fig.1), the SAW can be excited. If the illuminated area is small enough, it can be regarded as a point source. The displacement of SAW generated at the surface of a half-space or a plate was theoretically derived by Rose[5]. It is suitable to our case at room temperature while the PMMA can be considered as perfectly elastic solids. 
But what about that for the intermediate temperature range, it is instructive of the experimental work to investigate the SAW velocity of such materials.

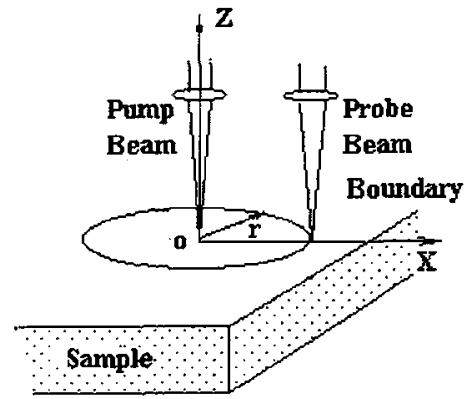

Fig.1 The mode of measurement
Several laser probes can be used to detect SAW. For knife-edge probe, the output signal $I_{\text {sig }}$ of the photodiode for detecting the acoustic travelling wave is given by[6]

$$
I_{s i g}=4 \pi \alpha(d / \Lambda)(\delta / \lambda) P_{0} \sin (\omega t-k x)
$$

where $\Lambda, \mathrm{k}, \mathrm{d}, \omega$ are the wavelength, wave number, displacement amplitude and angular frequency of the acoustic wave respectively, $\lambda$ is the optical wavelength, $\mathrm{d}$ is the diameter of the illuminated spot, $\mathrm{P}_{0}$ is the total incident optical power and $\alpha$ is the sensitivity of the photodiode. The SAW signals directly arrived from the source point and reflected from the boundary of the sample can be detected separately. When the time difference between the two pulse signals and the distance from the detection point to the boundary are measured precisely, the velocity of the SAW can be

determined.

When the temperature is not so high, then the viscoelastic properties of the sample do not change drastically, like our case, the spectrum approach[7] can be employed to evaluate the frequency dependence of the SAW phase velocity of PMMA. According to this method, the phase velocity of the ultrasonic pulse can be given by

$$
C(\omega)=\frac{\omega\left(X_{2}-X_{1}\right)}{\phi_{2}(\omega)-\phi_{1}(\omega)}
$$

where $\omega, X_{i}$, and $\phi_{i}(\omega)(i=1,2)$ are the angular frequency, the propagation distance and the phase at $X_{i}$ of acoustic pulse respectively. $\phi_{i}(\omega)$ can be evaluated through the FFT of the two pulse signais. In our work, the directly arrived SAW pulse signal and its reflection from boundary are used for the dispersion analysis of PMMA.

\section{EXPERIMENTAL METHOD}

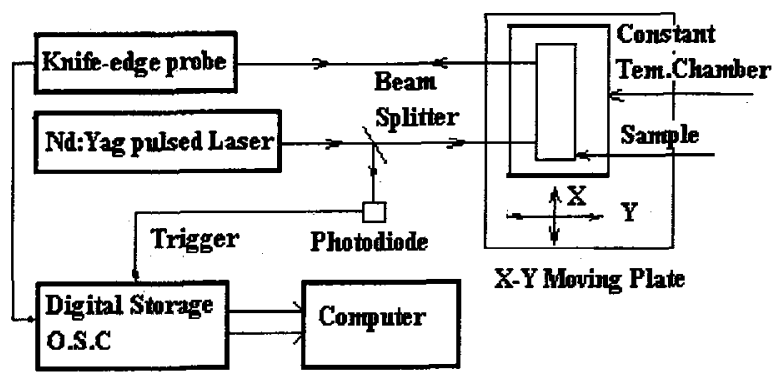

Fig.2 Schematic diagram of experimental setup
Fig. 2 shows the schematic diagram of the laser ultrasonic experimental system. A pulsed $\mathrm{Nd}$ :YAG laser working at the wavelength $532 \mathrm{~nm}$ with a $8 \mathrm{~ns}$ pulse duration and the energy up to $40 \mathrm{~mJ}$ is used as the exciting source of the pulsed ultrasonic wave. The laser beam is focused as small as possible on the surface of the sample. The power of the pump laser is adjusted enabling the doublepoled SAW waveform can be excited with point-source mode.

A knife-edge probe(home-

made) is used to detect the SAW. The output signal of the laser probe is fed into a digital storage O.S.C.(HP54510B) which is triggered by the laser pulse. Then the data is transferred to a microcomputer 


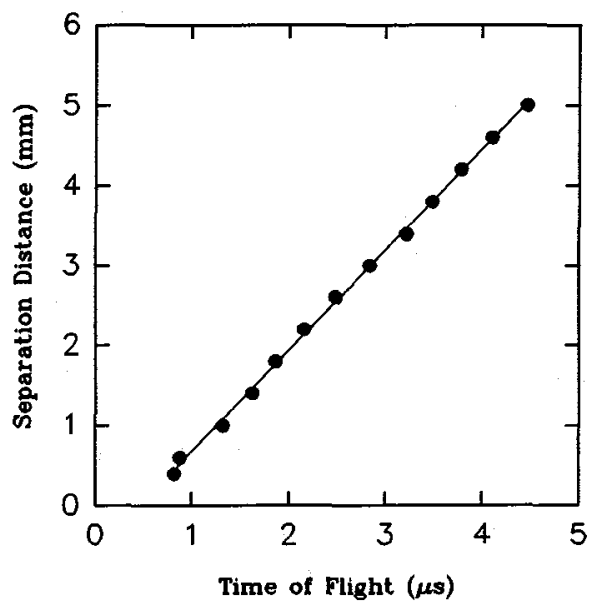

Fig. 3 Time of flight of SAW vase. separation distance for PMMA sample at room temperature.

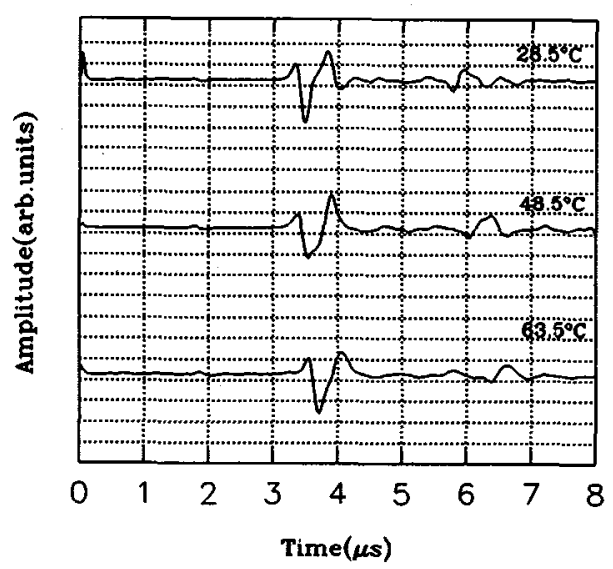

Fig. 4 Waveforms of SAW of PMMA sample at 28.5, 48.5 and $63.5^{\circ} \mathrm{C}$ respectively.

for further processing. The PMMA sample with the thickness of $6.7 \mathrm{~mm}$ or $4.8 \mathrm{~mm}$ is placed in a constant temperature chamber which is fixed on a X-Y moving stage. On the surface of the sample a thin $\mathrm{Al}$ film is deposited to ensure a good optic-reflective surface. The SAW velocities at different temperatures can be measured when the temperature of the constant-temperature chamber is changed. Thus we can get the relationship of the SAW velocity to temperature of PMMA. Using the Eq.(2), the dispersive properties of SAW for PMMA sample at different temperatures can also be analyzed.

\section{RESULTS AND ANALYSIS}

First, we measure the SAW velocity of PMMA at room temperature $\left(25^{\circ} \mathrm{C}\right)$. Fig. 3 gives the variation of the distance interval $\Delta \mathrm{X}$ versus the time of flight $\Delta \mathrm{t}$ for the SAW at room temperature. The distance interval is changed by moving the sample along the $X$ direction while the pump and probe laser beams are fixed. From Fig.3, we can see that the relation of $\Delta \mathrm{X}$ to $\Delta t$ of SAW for PMMA is linear and the

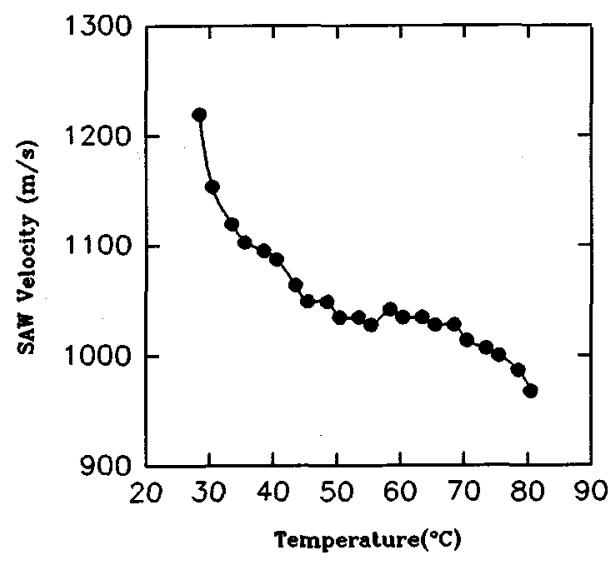

Fig.5 Temperature dependence of SAW velocity for PMMA

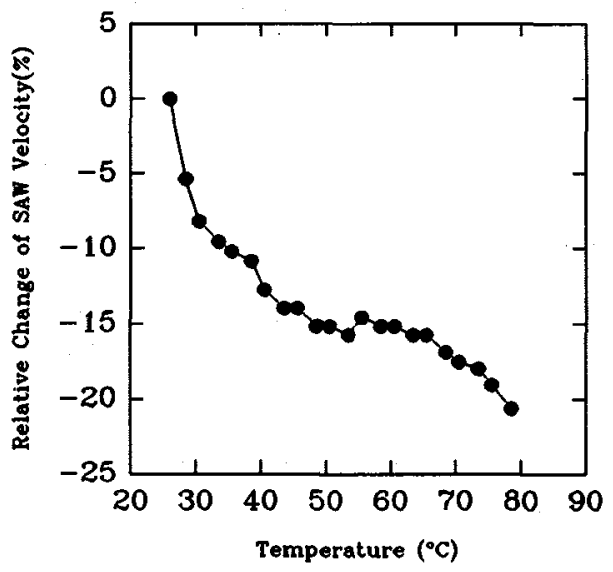

Fig. 6 Temperature dependence of change ratio of SAW velocity for PMMA. 


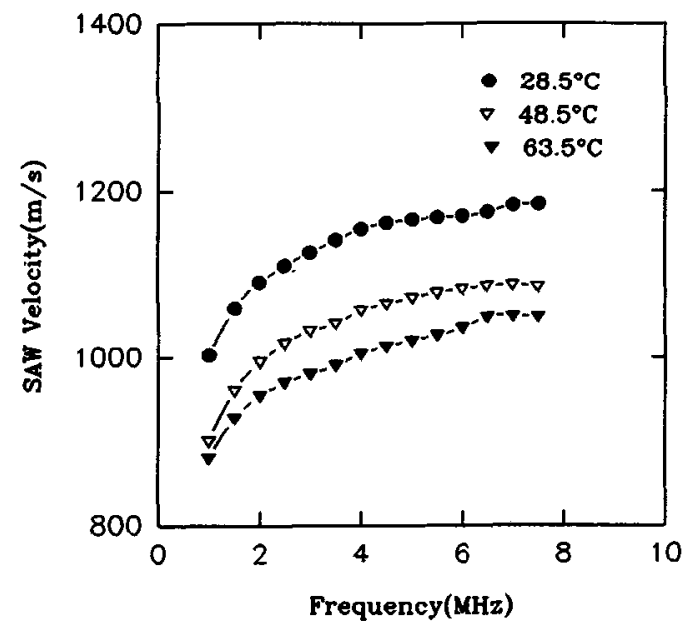

Fig.7 The SAW velocities as a function of frequency for PMMA at $28.5,48.5$ and $63.5^{\circ} \mathrm{C}$. slope of the curve is the SAW velocity which is $1250 \mathrm{~m} / \mathrm{s}$ in the experiment.

Then we measure the variation of SAW velocities of the samples versus temperature. Fig. 4 shows the experimental waveforms at three different temperatures $28.5,48.5$ and $63.5^{\circ} \mathrm{C}$. It is obvious that as the temperature increasing, the time interval between the two SAW signals increases, namely, the velocity decreases. The experimental results of the velocity and the relative change of the SAW velocity versus temperature are shown in Fig.5 and Fig.6 respectively. Both figures show that on the curve of velocity or relative change of velocity vs. temperature, there are two turning points, one of which is at $35^{\circ} \mathrm{C}$, and the other is near $50^{\circ} \mathrm{C}$ and extends to $68^{\circ} \mathrm{C}$. The first turning point may be related to the $\beta$-transition of PMMA, which is similar to the results of bulk waves[8]. The other turning point and extended area need to be further investigated.

Finally, the dispersion of SAW for PMMA is analyzed. When the data is processed, a Black-mann window[9] is used to minimize the effect of the time truncation on the frequency domain. The result is given in Fig.7. It is clear that the velocity of SAW for PMMA is frequency dependent and as the frequency increasing it becomes larger, while as the temperature rising, it becomes smaller. A wider bandwidth investigation may show the velocity peaks due to the relaxation of PMMA.

\section{CONCLUSIONS}

It has been demonstrated that a laser ultrasonic system with optic generation and detection SAW at the surface of a sample is a powerful tool to study the SAW properties for polymers. In this paper, the SAW velocity for PMMA as the functions of temperature and frequency is measured. The turning point of the SAW velocity near $35^{\circ} \mathrm{C}$ due to the $\beta$-transition is observed and the dispersion of SAW is analyzed. Further investigations including the measurement of the SAW attenuation and the study of the behaviour of SAW for PMMA in a wider frequency range are in progress.

\section{ACKNOWLEDGEMENTS}

This work is supported by the National Natural Science Foundation of China No.19134040.

\section{REFERENCES}

[1] I.L.Hopkins and C.R.Kurkjia, Physical Acoustics Principles and Methods, edt. by W.P.Mason (Academic, N.Y.), Vol II-part B(1964)102.

[2] B.Hertman and J.Jarzynbski., J.Appl.Phys. 43(11)(1972)4304.

[3] O.S.Lee, Materials Evaluation(1991)351.

[4] X.R.Zhang and C.M.Gan, Appl.Laser.China 7(6)(1987)246.

[5] L.R.F Rose, J.Acoust.Soc.Am.75(3),(1984)723-732.

[6] R.L.Whitman and A.Korpel, Applied Optics 8(8)(1969)1567.

[7] W.Sachse and Y.-H Pao, J.Appl.Phys 49(1978)4320-4327.

[8] X.R.Zhang, C.M.Gan, D.Fei, and S.Y.Zhang, Review of Progress in QNDE, Vol.13 eds. by D.O.Thompson et.al., (Plenum Publishing Corp.,1993)525-532.

[9] J.-D.Aussel and J.-P.Monchalin, Ultrasonics Vol.27(1989)165-177. 